\title{
New frontiers in human assisted reproduction - from research to clinical practice: Several considerations (Review)
}

\author{
SALVATORE GIZZO $^{1}$, MARCO NOVENTA ${ }^{1}$, MICHELA QUARANTA ${ }^{2}$, ROBERTA VENTURELLA ${ }^{3}$, \\ AMERIGO VITAGLIANO $^{1}$, MICHELE GANGEMI $^{1}$ and DONATO D'ANTONA ${ }^{1}$ \\ ${ }^{1}$ Department of Woman and Child Health-University of Padova, I-35128 Padova; \\ ${ }^{2}$ Department of Obstetrics and Gynaecology, University of Verona, I-37121 Verona; \\ ${ }^{3}$ Department of Obstetrics \& Gynaecology, 'Magna Graecia' University, I-88100 Catanzaro, Italy
}

Received September 22, 2015; Accepted August 22, 2016

DOI: $10.3892 / \mathrm{mmr} .2016 .5749$

\begin{abstract}
In the era of very late, or advanced, motherhood, in which 'egg banks', 'social' egg-freezing, egg donation and surrogacy represent a potential solution to a number of obstacles to human reproduction, what is the role of scientists and clinicians involved in assisted reproduction? In light of the apprehension that, in the future, through fertility treatment infertility may be passed on to the offspring, boundaries of medical vs. 'social' infertility are being created. Scientists and clinicians are joining forces in a synergistic effort to improve the effectiveness of infertility care by introducing novel therapeutic protocols with the intent of customising care and improving cost-effectiveness, testing novel drugs and formulations, and searching for novel markers (for estimating biological age) and nomograms (to optimise the yield of a controlled ovarian hyperstimulation cycle). On the other hand, political, social and health institutions are doing little to educate young women with respect to disinformation and to increase their awareness regarding age as the predominant factor that contributes towards the decline in fertility. Nevertheless, despite the great advances that have been made, 38 years after the birth of the first baby via in vitro fertilisation, the intricate road leading from the antral follicle to the fully developed baby continues to be designated as being too 'expensive',
\end{abstract}

Correspondence to: Professor Salvatore Gizzo, Department of Woman and Child Health-University of Padova, Via Giustiniani 3, I-35128 Padova, Italy

E-mail: ginecologia_padova@libero.it

Abbreviations: ARTs, assisted reproductive technologies; COS, controlled ovarian hyperstimulation; HPV, human papilloma virus; IVF, in vitro fertilisation; PGD, pre-implantation genetic diagnosis; PGS, pre-implantation genetic screening; TLM, time-lapse monitoring

Key words: assisted reproduction, biotechnology, cost-effectiveness, counselling, ethics, genetic screening, health care policy, pre-implantation genetic screening, metabolomics, morphokinetic, embryos 'empirical', 'mysterious' or 'bound by ethics', with few significant improvements in terms of real cost-effectiveness.

\section{Contents}

1. Introduction

2. Critical overview of the state of the art

3. Concluding remarks

\section{Introduction}

In an era of advanced, or very late, motherhood, strategies including 'social' egg-freezing, surrogacy and commercialisation of fertility treatments represent a potential solution to overcoming obstacles to human reproduction, while simultaneously turning infertile couples into potential consumers. Leaving aside the potential ethical and obstetrical problems associated with an advanced maternal age [as consequences of in vitro fertilisation (IVF) and, in particular, of heterologous fertilisation], which certainly warrant further investigation (1-4), if assisted reproduction technologies may be considered as a 'pendulum swinging' between the health care service and industry in offering solutions against time, what then are the roles to be adopted by scientists and clinicians involved in human reproductive care? What are their responsibilities, and what effects will developments in the future have?

Caution must always be exercised in the interpretation of evidence generated by investigations on infertility care to avoid the generation of 'speculative' fears, as, for example, in the case of the hypothetical 'transmission of infertility to the offspring' of fetuses grown in infertile couples who underwent IVF treatments (5). Nevertheless, in developed countries, the increasing prevalence of infertility warrants a further investigation of the mechanisms that are involved, and rigorous boundaries between medical vs. social infertility, which are gradually being created, will be of vital importance in the near future. Scientists and clinicians are joining forces in a synergistic effort to improve the effectiveness of infertility care by introducing novel therapeutic protocols for the purpose of customising care and improving cost-effectiveness, for example, by adopting 
different formulations of gonadotropins according to different cohorts of patients and the IVF setting $(6,7)$, testing novel drugs (for example, introducing recombinant luteinising hormone) and formulations (e.g. long-acting gonadotropins), and searching for novel markers for estimating biological age $(8,9)$, and novel nomograms to optimise the yield of a controlled ovarian hyper-stimulation cycle. On the other hand, political, social and health institutions are doing little to educate young women with respect to disinformation and to increase their awareness regarding age as the predominant factor that contributes towards the decline in fertility.

\section{Critical overview of the state of the art}

In developed countries, the number of couples referred for assisted reproduction due to age-associated infertility is growing exponentially due to career priorities, financial concerns and the increasing trend in postponing marriage and childbearing (10).

It remains to be demonstrated whether increased awareness of the interplay between age and fertility should lead to a lesser degree of postponement of motherhood, since the question of a woman's 'biological clock' is, indeed, an important sociopolitical issue that is influenced by several broader factors, among which are the rise in higher education among women, the demands of the labor market, technological developments and financial interests stemming from the 'reproduction industry'. However, even though the immense size of the problem could erroneously induce a sense of powerlessness in terms of finding a solution, it would appear to be mandatory to encourage educational efforts by schools, the health care system and the media to eradicate the fertility 'myth' (i.e. the mistaken belief that, in the field of reproduction, modern medicine is already able to overcome the barriers imposed by biological age), particularly among young people, as subsequently acting on the misinformation may result in a reliance on costly, unpleasant techniques with potentially disappointing outcomes. In so doing, access to improved biological/medical information may indeed raise the awareness of the decline in age-associated fertility (11).

Nonetheless, despite the great advances that have been made, 38 years after the birth of the first IVF baby (Louise Brown on July 25, 1978), the intricate road leading from the antral follicle to the fully developed baby continues to be designated as being too 'expensive', 'empirical', 'mysterious' or 'bound by ethics', with few significant improvements in terms of real cost-effectiveness.

Regarding costs, rational intuition should lead us to reflect upon and act on the variable costs (predominantly due to drugs and stimulation protocols) rather than on fixed costs (attributable to clinicians, the laboratory and research). It is a concern that variable costs are frequently unduly influenced by a consideration of the time involved, or the 'inappropriateness' of the stimulation protocols.

In addition to the efforts made by scientists to increase the number and level of sophistication of treatment options so as to improve the personalisation of care and overall cost-effectiveness, clinicians should promote both primary (i.e. counselling regarding age-associated infertility) and secondary prevention (for the early diagnosis of disease potentially able to compromise and/or delay the search for a first pregnancy, including common female/male endocrine issues, immunological infective disorder or female specific fertility compromising disease like endometriosis, polycystic ovarian syndrome, uterine/tubal organic pathologies), improving the estimation of pretreatment prognoses in order to choose the most appropriate controlled ovarian stimulation (COS) protocols. Expanding research programs will be able, in the near future, to resolve a large proportion of the confusion regarding the empirical prescription of drugs with orphan designations in IVF, whose positive (or maybe, negative) effects have yet to be confirmed from either the clinical and/or the molecular point of view (12-15). Certainly, the empirical approach will never be eradicated from clinical practice, at least, not prior to the clarification of certain myths, mysteries and misconceptions $(16,17)$. It remains our conviction that scientists, with their novel discoveries, should be called upon first of all to solve the problems associated with empirical treatments. However, research requires a level of economic investment that is rarely made in low-cost-treatment infertility centres, which therefore prolongs the application of the empirical approach in the clinic, and the negative consequences that may ensue from this.

Selecting one example as a suitable allegory, the sheer quantity of speculation regarding luteal phase support and endometrial receptivity following cycles of IVF (both fresh and thawed) (18), and the molecular interactions that are formed between the blastocyst and the endometrium following embryo transfer, may be compared to our understanding of Egyptian hieroglyphs prior to Jean-François Champollion's studies on the Rosetta Stone. While the sheer availability of resources would prevent the publication of a voluminous scientific literature on the topic, further experimental research is required prior to beginning clinical trials. Furthermore, in approaching experimental questions on human gametes and embryos from the perspective of performing the basic research, scientists may be confronted by the 'gatekeepers' or 'custodians' of ethics, who place huge obstacles in their path, as is the case, for example, with research on genetic pre-implantation in numerous countries (19).

These limitations may be partly considered as the driving forces behind the provision of anachronistic misinformation and general ignorance regarding topics of concern, including human papilloma virus (HPV) infection and idiopathic infertility $(20,21)$, fertility preservation in patients suffering from malignancy $(22,23)$ and the applicability of gonadal stem cells in infertility (24), issues which may potentially represent the future frontier of human reproductive medicine. Nevertheless, even if, in certain situations, ethics may be a barrier, modern scientists and those of the future will be dutifully obligated to navigate a way through, rather than avoid, these issues. Despite the fact that the solution to this dilemma may be less simple than it appears, it may be asserted that, in the era of '-omics', it is not an arduous task to circumvent the obstacles. Since the inception of IVF, selection of the most competent embryos for transfer has been a primary focus of investigation. As the field has progressed, an increasing number of studies have concentrated on developing more advanced technologies, invasive [such as pre-implantation genetic diagnosis (PGD) and pre-implantation genetic screening (PGS)] and non-invasive [such as time-lapse monitoring (TLM) and metabolomics/proteomic approaches], to select the most 
competent embryos with the highest potential of implantation for transfer $(25,26)$.

Political scientists, doctors and bioethics experts have discussed the need for governments to improve the regulation of research and the clinical use of ARTs (assisted reproductive technologies). Certain experts and stakeholders argue for the adoption of novel legislation that will allow scientists to achieve the potential benefits of reproductive technologies that may be applicable to human health. Conversely, others see legislation as necessary to prevent scientific exploration into ethically unacceptable areas. Others again, of course, question the need for any government involvement in this regard (27).

A clear example of the existing confusion may be seen in the application of PGD, a technique currently performed at the blastocyst stage as PGS that was originally developed as an alternative approach to prenatal diagnosis for couples who present a high risk of transmitting a genetic defect, and which has been subsequently confirmed as a useful technology for improving the cost-effectiveness of IVF (28).

Concerning PGD, complex issues associated with ethics and equitable access to embryonic genetic testing have become even more complicated and controversial in legislative debates. Although several initiatives have attempted to harmonise legislation across Europe and intercontinentally, a diversity of health care systems and the presence of cultural differences have hampered attempts to achieve this goal (28).

Thus, in each country, PGD is used with a specific approach that should reflect the views held by scientific groups, professional societies, legislators and society itself on the appropriate use of this technology. Although several countries have banned the use of PGD, others are discussing novel applications and regulatory strategies of this technology in order to maintain the method's reliability by defining standards and responsibilities for professionals performing PGD, and by protecting the rights of those involved. In other countries, there are no direct regulations for the technique, and, instead, professional guidelines for practicing service providers are consulted (29).

Those involved in ARTs have tried to solve the existing disparities between the potential capability of technologies and their clinical applicability by drawing from past experience in the use of non-invasive technologies. As a result, TLM has been implemented in numerous clinics worldwide. The proposed benefits of the method when compared with culture in a standard incubator and fixed time-point evaluation include uninterrupted culture, a flexible workflow in the laboratory and improved embryo selection. The latter is based on the reasonable assumption that more frequent observations will provide substantially more information on the association of development, timing and embryo viability. Although several retrospective studies $(30,31)$ have confirmed an association between TLM parameters and embryo viability evaluated by developmental competence, aneuploidy and clinical pregnancy, TLM should prove to be useful for laboratories specialising in IVF in prospective randomised studies. However, given the relatively high expenses associated with acquiring an instrument and the ancillary products, the verification of the cost-effectiveness of TLM will be a matter of future developments, competition and the judgment of the individual clinics (32).

It is quite likely that, in the near future, TLM may generate promising results if it is associated with PGS, a technology that offers the advantage of biopsy of the blastocyst trophectoderm on day 5 or 6 .

The introduction of PGS opens up the era of 'delayed' genetic investigations: Firstly, a greater amount of genetic material may be retrieved from biopsies of blastocysts compared with embryos at the cleavage stage (i.e. a greater number of cells facilitates genetic analysis, providing more accurate results, which thereby enables an easier detection of genetic and chromosomal abnormalities). Secondly, biopsy of the trophectoderm on day 5 post-fertilisation involves embryos that have successfully negotiated the initial steps of cell differentiation, and for this reason these embryos have the highest implantation potential. Thirdly, several recent studies $(33,34)$ have also shown that the rate of aneuploidy is markedly lower in blastocysts compared with cleavage-stage embryos, and finally, biopsies performed on cleavage-stage embryos are more likely to be damaging compared with those performed on blastocysts (35).

On the one hand, the progress made in genomic research (whole-genome sequencing) may lead to whole-genome analysis and the potential for the diagnosis of diseases for which the genetic background has not yet been (or is insufficiently well) elucidated; on the other hand, the lack of well-defined and globally accepted guidelines regarding the application of genome-wide diagnostic testing in IVF, in addition to the heterogeneity of the numerous figures involved (clinicians, scientists, jurists, ethicists, patients, organisations and policy makers), may increase the complexity of the issues that are prolonging the debate. However, all is not lost, since in this progressive era of '-omics', the option of acquiring genetic information without direct genome analysis is now a concrete possibility, and this could be applicable in the clinical practice.

Metabolic profiling, or metabolomics, is the analysis of various molecular metabolites within cells and fluids through the use of various forms of spectral and analytical approaches, and it attempts to determine the metabolites associated with physiological and pathological states. It offers a significant advantage over the use of genomic, transcriptomic and proteomic approaches. Smaller variations in gene expression and protein synthesis result in an amplified change in the metabolite profile, known as the 'metabolome', and this information may be used to detect subtle cellular events (36).

Metabolic turnover is crucial for a pre-implantation embryo to grow and result in a successful pregnancy. Accordingly, preliminary studies and pioneering investigations into the nutrients and metabolites present within culture media and biological fluids (blood, urine, follicular fluid and endometrial supernatant) are yielding fascinating results, which are likely to ensure that this candidate technology is a most promising tool for predicting embryo quality without invasiveness $(37,38)$.

An understanding of the early effects of genetic abnormalities through a metabolomics approach may allow us to collect, during the pre-implantation stage and perhaps in the pre-fertilisation period, more accurate information without the invasiveness and the ethical barriers pertaining to current techniques (39-41).

\section{Concluding remarks}

Ultimately, the key message for readers of the present review, and those with a keen vested interest in this area, would be to 
offer encouragement to produce more compelling evidence based on novel discoveries obtained by rigorous investigations in order to improve the overall success of infertility care. Only a correct methodology in approaching this topic may eradicate several myths and misconceptions, solve a large part of the dilemma and propose novel treatments that would be able to really improve the prospects for 'infertility care users' (42-44).

While fully sharing Einstein's thought that 'we cannot solve the problems with the same thinking we used when we created them' (45), it is our considered opinion that often problems do not require a solution to solve them; instead, they require maturity to outgrow them.

\section{Acknowledgements}

We would like to thank the whole team of the Assisted Reproduction Unit of the University of Padova.

\section{References}

1. Favilli A, Pericoli S, Acanfora MM, Bini V, Di Renzo GC and Gerli S: Pregnancy outcome in women aged 40 years or more. J Matern Fetal Neonatal Med 25: 1260-12633, 2012.

2. Favilli A, Acanfora MM, Bini V, Radicchi R, Di Renzo GC and Gerli S: Single indication of labor induction with prostaglandins: Is advanced maternal age a risk factor for cesarean section? A matched retrospective cohort study. J Matern Fetal Neonatal Med 26: 665-6688, 2013.

3. Gizzo S, Quaranta M, Andrisani A, Bordin L, Vitagliano A, Esposito F, Venturella R, Zicchina C, Gangemi M and Noventa M: Serum stem cell factor assay in elderly poor responder patients undergoing IVF: A new biomarker to customize follicle aspiration cycle by cycle. Reprod Sci 23: 61-68, 2016.

4. Gizzo S, Andrisani A, Noventa M, Manfè S, Oliva A, Gangemi M, Nardelli GB and Ambrosini G: Recombinant LH supplementation during IVF cycles with a GnRH-antagonist in estimated poor responders: A cross-matched pilot investigation of the optimal daily dose and timing. Mol Med Rep 12: 4219-4229, 2015.

5. Díaz-García C, Estella C, Perales-Puchalt A and Simón C: Reproductive medicine and inheritance of infertility by offspring: The role of fetal programming. Fertil Steril 96: 536-545, 2011

6. Gerli S, Bini V, Favilli A and Di Renzo GC: Clinical efficacy and cost-effectiveness of HP-human FSH (Fostimon $\left.{ }^{\circledR}\right)$ versus rFSH (Gonal-F®) in IVF-ICSI cycles: A meta-analysis. Gynecol Endocrinol 29: 520-529, 2013.

7. Ripellino C, Visentin E, Gizzo S and Bühler K: A Cost-effectiveness evaluation comparing biosimilar bemfola to Gonal-F for the treatment of infertility in an Italian contest. Value Health 18: A735, 2015.

8. Gizzo S, Andrisani A, Noventa M, Quaranta M, Esposito F, Armanini D, Gangemi M, Nardelli GB, Litta P, D'Antona D and Ambrosini G: Menstrual cycle length: A surrogate measure of reproductive health capable of improving the accuracy of biochemical/sonographical ovarian reserve test in estimating the reproductive chances of women referred to ART. Reprod Biol Endocrinol 13: 28, 2015.

9. Venturella R, Lico D, Sarica A, Falbo MP, Gulletta E, Morelli M, Zupi E, Cevenini G, Cannataro M and Zullo F: OvAge: A new methodology to quantify ovarian reserve combining clinical, biochemical and 3D-ultrasonographic parameters. J Ovarian Res 8: 21, 2015.

10. Gizzo S, Andrisani A, Esposito F, Oliva A, Zicchina C, Capuzzo D, Gangemi M and Nardelli GB: Ovarian reserve test: An impartial means to resolve the mismatch between chronological and biological age in the assessment of female reproductive chances. Reprod Sci 21: 632-639, 2014.

11. Hashiloni-Dolev Y, Kaplan A and Shkedi-Rafid S: The fertility myth: Israeli students' knowledge regarding age-related fertility decline and late pregnancies in an era of assisted reproduction technology. Hum Reprod 26: 3045-3053, 2011.

12. Vitagliano A, Quaranta M, Noventa M and Gizzo S: 'Empiric' inositol supplementation in normal-weight non insulin resistant women with polycystic ovarian disease: From the absence of benefit to the potential adverse effects. Arch Gynecol Obstet 291: 955-957, 2015
13. Gizzo S, Capuzzo D, Zicchina C, Di Gangi S, Coronella ML, Andrisani A, Gangemi M and Nardelli GB: Could empirical low-dose-aspirin administration during IVF cycle affect both the oocytes and embryos quality via COX 1-2 activity inhibition? J Assist Reprod Genet 31: 261-268, 2014.

14. Vitagliano A, Noventa M and Gizzo S: Is it time to consider patients suffering from endometriosis-related infertility as 'novel candidates' for targeted peri-conceptional D-chiro inositol supplementation? Hypothesis, rationale and some considerations. J Assist Reprod Genet 32: 407-408, 2015

15. Vitagliano A, Noventa $M$ and Gizzo S: Emerging evidence regarding statins use as novel endometriosis targeted treatment: Real 'magic pills' or 'trendy' drugs? Some considerations. Eur J Obstet Gynecol Reprod Biol 184: 125-126, 2015.

16. Ghirardello A, Gizzo S, Noventa M, Quaranta M, Vitagliano A, Gallo N, Pantano G, Beggio M, Cosma C, Gangemi M, et al: Acute immunomodulatory changes during controlled ovarian stimulation: Evidence from the first trial investigating the short-term effects of estradiol on biomarkers and B cells involved in autoimmunity. J Assist Reprod Genet, 2015. (Epub ahead of print)

17. Gizzo S, Noventa M, Quaranta M, Vitagliano A, Esposito F, Andrisani A, Venturella R, Alviggi C, Plebani M, Gangemi M, et al: The potential role of GnRH agonists and antagonists in inducing thyroid physiopathological changes during IVF. Reprod Sci, 2015. (Epub ahead of print)

18. Gizzo S, Andrisani A, Esposito F, Noventa M, Di Gangi S, Angioni S, Litta P, Gangemi M and Nardelli GB: Which luteal phase support is better for each IVF stimulation protocol to achieve the highest pregnancy rate? A superiority randomized clinical trial. Gynecol Endocrinol 30: 902-908, 2014.

19. Carter D and Braunack-Mayer A: The appeal to nature implicit in certain restrictions on public funding for assisted reproductive technology. Bioethics 25: 463-471, 2011.

20. Noventa M, Andrisani A, Gizzo S, Nardelli GB and Ambrosini G: Is it time to shift the attention on early stages embryo development to avoid inconclusive evidence on HPV-related infertility: Debate and proposal. Reprod Biol Endocrinol 12: 48, 2014.

21. Gizzo S, Ferrari B, Noventa M, Ferrari E, Patrelli TS, Gangemi M and Nardelli GB: Male and couple fertility impairment due to HPV-DNA sperm infection: Update on molecular mechanism and clinical impact-systematic review. Biomed Res Int 2014: 230263, 2014.

22. Practice Committee of American Society for Reproductive Medicine: Ovarian tissue cryopreservation: A committee opinion. Fertil Steril 101: 1237-1243, 2014.

23. Gizzo S, Ancona E, Patrelli TS, Saccardi C, Anis O, Donato D and Nardelli GB: Fertility preservation in young women with cervical cancer: An oncologic dilemma or a new conception of fertility sparing surgery? Cancer Invest 31: 189, 2013.

24. Albertini DF: Twists and turns in the business of gonadal stem cells. J Assist Reprod Genet 31: 933-934, 2014.

25. Yang Z, Zhang J, Salem SA, Liu X, Kuang Y, Salem RD and Liu J: Selection of competent blastocysts for transfer by combining time-lapse monitoring and array $\mathrm{CGH}$ testing for patients undergoing preimplantation genetic screening: A prospective study with sibling oocytes. BMC Med Genomics 7: 38, 2014

26. Ciray HN, Campbell A, Agerholm IE, Aguilar J, Chamayou S, Esbert M and Sayed S; Time-Lapse User, Group: Proposed guidelines on the nomenclature and annotation of dynamic human embryo monitoring by a time-lapse user group. Hum Reprod 29: 2650-2660, 2014.

27. Damian BB, Bonetti TC and Horovitz DD: Practices and ethical concerns regarding preimplantation diagnosis. Who regulates preimplantation genetic diagnosis in Brazil? Braz J Med Biol Res 48: 25-33, 2015.

28. Knoppers BM, Bordet S and Isasi RM: Preimplantation genetic diagnosis: An overview of socio-ethical and legal considerations. Annu Rev Genomics Hum Genet 7: 201-221, 2006.

29. Harton GL, Magli MC, Lundin K, Montag M, Lemmen J and Harper JC; European Society for Human Reproduction and Embryology (ESHRE) PGD Consortium/Embryology Special Interest Group: ESHRE PGD consortium/embryology special interest group-best practice guidelines for polar body and embryo biopsy for preimplantation genetic diagnosis/screening (PGD/PGS). Hum Reprod 26: 41-46, 2011.

30. Aparicio-Ruiz B, Basile N, Pérez Albalá S, Bronet F, Remohí J and Meseguer M: Automatic time-lapse instrument is superior to single-point morphology observation for selecting viable embryos: retrospective study in oocyte donation. Fertil Steril: 2016. 
31. Motato Y, de los Santos MJ, Escriba MJ, Ruiz BA, Remohí J and Meseguer M: Morphokinetic analysis and embryonic prediction for blastocyst formation through an integrated time-lapse system. Fertil Steril 105: 376-384, 2016.

32. Kirkegaard K, Ahlström A, Ingerslev HJ and Hardarson T: Choosing the best embryo by time lapse versus standard morphology. Fertil Steril 103: 323-332, 2015.

33. Scott RT Jr, Upham KM, Forman EJ, Zhao T and Treff NR: Cleavage-stage biopsy significantly impairs human embryonic implantation potential while blastocyst biopsy does not: a randomized and paired clinical trial. Fertil Steril 100: 624-630, 2013.

34. Cimadomo D, Capalbo A, Ubaldi FM, Scarica C, Palagiano A, Canipari R and Rienzi L: The Impact of Biopsy on Human Embryo Developmental Potential during Preimplantation Genetic Diagnosis. Biomed Res Int: 2016.

35. Zakharova EE, Zaletova VV and Krivokharchenko AS: Biopsy of human morula-stage embryos: Outcome of 215 IVF/ICSI cycles with PGS. PLoS One 9: e106433, 2014.

36. Egea RR, Puchalt NG, Escrivá MM and Varghese AC: OMICS: Current and future perspectives in reproductive medicine and technology. J Hum Reprod Sci 7: 73-92, 2014.

37. Leese HJ: Metabolism of the preimplantation embryo: 40 years on. Reproduction 143: 417-427, 2012.

38. Wallace M, Cottell E, Gibney MJ, McAuliffe FM, Wingfield M and Brennan L: An investigation into the relationship between the metabolic profile of follicular fluid, oocyte developmental potential, and implantation outcome. Fertil Steril 97: 1078-1084. e1-8, 2012
39. Zuradzki T: A situation of ethical limbo and preimplantation genetic diagnosis. J Med Ethics 40: 780-781, 2014.

40. Hens K, Dondorp W, Handyside AH, Harper J, Newson AJ, Pennings G, Rehmann-Sutter C and de Wert G: Dynamics and ethics of comprehensive preimplantation genetic testing: A review of the challenges. Hum Reprod Update 19: 366-375, 2013.

41. Rubio I, Galán A, Larreategui Z, Ayerdi F, Bellver J, Herrero J and Meseguer M: Clinical validation of embryo culture and selection by morphokinetic analysis: A randomized, controlled trial of the EmbryoScope. Fertil Steril, 2014.

42. Venturella R, Morelli M, Lico D, Di Cello A, Rocca M, Sacchinelli A, Mocciaro R, D'Alessandro P, Maiorana A, Gizzo S and Zullo F: Wide excision of soft tissues adjacent to the ovary and fallopian tube does not impair the ovarian reserve in women undergoing prophylactic bilateral salpingectomy: Results from a randomized, controlled trial. Fertil Steril 104: 1332-1339, 2015

43. Gizzo S, Vitagliano A, Noventa M, Litta P, Saccardi C and Quaranta M: Surgery, endometriosis-related infertility and negative impact on ovarian reserve: 'Which came first, the hen or the egg?' An unresolved dilemma. Arch Gynecol Obstet 292: 709-711, 2015.

44. Andrisani A, Donà G, Tibaldi E, Brunati AM, Sabbadin C, Armanini D, Alvisi G, Gizzo S, Ambrosini G, Ragazzi E and Bordin L: Astaxanthin improves human sperm capacitation by inducing Lyn displacement and activation. Mar Drugs 13: 5533-5551, 2015.

45. Einstein A: Atomic Education Urged by Einstein. New York Times, 25 May 1946. 